\title{
The Clinical and Molecular Epidemiology of CTX-M-9 Group Producing Enterobacteriaceae Infections in Children
}

\author{
Latania K. Logan (D) - Rachel L. Medernach · T. Nicholas Domitrovic · Jared R. Rispens • \\ Andrea M. Hujer · Nadia K. Qureshi · Steven H. Marshall • David C. Nguyen · Susan D. Rudin • \\ Xiaotian Zheng $\cdot$ Sreenivas Konda $\cdot$ Robert A. Weinstein · Robert A. Bonomo
}

Received: December 10, 2018 / Published online: February 16, 2019

(C) The Author(s) 2019

\section{ABSTRACT}

Introduction: The pandemic of extended-spectrum beta-lactamase-(ESBL)-producing Enterobacteriaceae (Ent) is strongly linked to the dissemination of CTX-M-type-ESBL-Ent. We sought to define the epidemiology of infections in children due to an emerging resistance type,

Enhanced digital features To view enhanced digital features for this article go to https://doi.org/10.6084/ m9.figshare.7660973.

L. K. Logan $(\varangle)$. R. L. Medernach .

J. R. Rispens · D. C. Nguyen

Pediatrics, Rush University Medical Center, Rush

Medical College, Chicago, IL, USA

e-mail: Latania_Logan@rush.edu

L. K. Logan · T. N. Domitrovic · A. M. Hujer

S. H. Marshall - S. D. Rudin - R. A. Bonomo

Research Service, Louis Stokes Cleveland

Department of Veterans Affairs Medical Center,

Cleveland, $\mathrm{OH}$, USA

T. N. Domitrovic · A. M. Hujer · D. C. Nguyen ·

R. A. Bonomo

Case Western Reserve School of Medicine,

Cleveland, $\mathrm{OH}, \mathrm{USA}$

R. A. Bonomo

Pharmacology, Case Western Reserve University

School of Medicine, and the CWRU-Cleveland

VAMC Center for Antimicrobial Resistance and Epidemiology (Case VA CARES), Cleveland, OH, USA
CTX-M-9-group-producing-Ent (CTX-M-9-grpEnt).

Methods: A retrospective matched case-control analysis of children with CTX-M-9-grp-Ent infections who received medical care at three Chicago area hospitals was performed. Cases were defined as children possessing extendedspectrum cephalosporin-resistant (ESC-R)

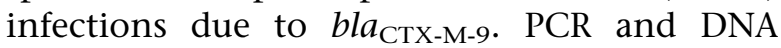
analysis assessed beta-lactamase (bla) genes, multi-locus sequence types (MLST) and phylogenetic grouping of $E$. coli. Controls were children with ESC-susceptible (ESC-S)-Ent infections matched one case to three controls

\section{R. A. Bonomo}

Molecular Biology and Microbiology, Case Western Reserve University School of Medicine, and the CWRU-Cleveland VAMC Center for Antimicrobial Resistance and Epidemiology (Case VA CARES), Cleveland, $\mathrm{OH}$, USA

\section{R. A. Bonomo}

Biochemistry, Case Western Reserve University School of Medicine, and the CWRU-Cleveland VAMC Center for Antimicrobial Resistance and Epidemiology (Case VA CARES), Cleveland, $\mathrm{OH}$, USA

\section{R. A. Bonomo}

Proteomics and Bioinformatics, Case Western Reserve University School of Medicine, and the CWRU-Cleveland VAMC Center for Antimicrobial Resistance and Epidemiology (Case VA CARES), Cleveland, $\mathrm{OH}$, USA 
by age, source, and hospital. The clinical-epidemiologic predictors of CTX-M-9-grp-Ent infection were assessed.

Results: Of 356 ESC-R-Ent isolates from children (median age 4.1 years), the CTX-M-9group was the solely detected bla gene in 44 (12.4\%). The predominant species was E. coli (91\%) of virulent phylogroups D (60\%) and B2 (40\%). MLST revealed multiple strain types. On multivariable analysis, CTX-M-9-grp-Ent occurred more often in E. coli than other Ent genera (OR 7.4, 95\% CI 2.4, 27.2), children of nonBlack-White-Hispanic race (OR 7.4, 95\% CI 2.4, 28.2), and outpatients (OR 4.5, 95\% CI 1.7, 12.3), which was a very unexpected finding for infections due to antibiotic-resistant bacteria. Residents of South Chicago had a 6.7 times higher odds of having CTX-M-9-grp-Ent infections than those in the reference region (West), while residence in Northwestern Chicago was associated with an $81 \%$ decreased odds of infection. Other demographic, comorbidity, invasive-device, and antibiotic use differences were not found.

Conclusion: CTX-M-9-grp-Ent infection may be associated with patient residence and is occurring in children without traditional in-patient exposure risk factors. This suggests that among children, the community environment may be

X. Zheng

Microbiology, Ann and Robert H. Lurie Children's

Hospital of Chicago, Chicago, IL, USA

X. Zheng

Pathology, Northwestern University Feinberg

School of Medicine, Chicago, IL, USA

N. K. Qureshi

Pediatrics, Loyola University Medical Center,

Maywood, IL, USA

R. L. Medernach · J. R. Rispens · D. C. Nguyen ·

R. A. Weinstein

Medicine, Rush University Medical Center, Rush

Medical College, Chicago, IL, USA

L. K. Logan · R. A. Weinstein

Cook County Health and Hospital Systems,

Chicago, IL, USA

S. Konda

Epidemiology and Biostatistics, University of Illinois at Chicago, Chicago, IL, USA a key contributor in the spread of these resistant pathogens.

Keywords: Children; Drug resistance; Enterobacteriaceae infections; Epidemiology; Gramnegative bacteria

\section{INTRODUCTION}

The pandemic of multi-drug-resistant (MDR) Enterobacteriaceae remains one of the most significant public health threats of our time $[1,2]$. MDR Enterobacteriaceae are associated with significant morbidity and mortality in infected individuals, and an increasing number of reports describe extra-intestinal and invasive infections with these organisms in children [3-5]. Many studies show that beta-lactamase (bla) genes harbored by Enterobacteriaceae, e.g., extended-spectrum beta-lactamases (ESBLs or $\left.b l a_{\mathrm{ESBLs}}\right)$ and carbapenemases (e.g., $\left.b{ }_{\mathrm{KPCs}}\right)$, are the major contributors to this growing problem $[6,7]$. Strains carrying bla genes often harbor additional antibiotic resistance determinants residing on mobile genetic elements, e.g., plasmids and transposons, which are capable of rapid dissemination [1, 7].

In the US and worldwide, the CTX-M-type ESBL-producing Enterobacteriaceae (CTX-M Ent) are the predominant ESBL-producing strains, with the clonal multi-locus sequence type (ST) 131 E. coli harboring CTX-M-15 (CTXM-1 group) being the most commonly reported in both adult and pediatric studies [7, 8]. However, several other CTX-M types continue to circulate in Ent and are equally concerning, highly resistant pathogens that can be acquired in the community [9].

A notable example is the widespread CTX-M9-group ESBL genes (е.g., $b l a_{\text {CTХ-М-9, }} b l a_{\text {CTX-M-14, }}$

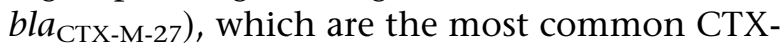
$\mathrm{M}$ genes circulating in Ent in some geographic regions [10]. A 2016 US study of the prevalence of CTX-M genes in Enterobacteriaceae found relative stability of $b{ } a_{\mathrm{CTX}-\mathrm{M}-15}$ in $E$. coli. Nevertheless, an increase in the prevalence of $b{ }_{a} a_{\text {СтX- }}$ M-14 in bla-producing E. coli occurred (20.4\% in 2014) compared with 2012 (16.0\%) [11]. Despite this, much less is reported concerning 
the epidemiology of CTX-M-9-group infections. Unlike the clonal ST131 E. coli strains, CTX-M9-group genes are associated with multiple strain types, and horizontal gene transfer via mobile genetic elements likely plays an important role in their dissemination [12]. While studies of ESBL-Ent in US children and internationally have reported infections due to CTXM-9-group-producing Enterobacteriaceae (CTXM-9-grp-Ent) [10, 13], pediatric studies have not focused on the clinical and molecular epidemiology and impact of infections specifically due to CTX-M-9-grp-Ent.

In previous investigations, the genetic basis for beta-lactam resistance in Enterobacteriaceae isolates recovered from children cared for by multiple centers in the Chicago area was determined [13]. Subsequently, subgroups of children with infections due to similar resistance determinants, (e.g., plasmid-mediated fluoroquinolone, ESBL, and carbapenem resistance genes) were analyzed to determine genotypes, host factors, and exposures associated with specific MDR Enterobacteriaceae strains $[13,14]$. An analysis of children with plasmidmediated fluoroquinolone-resistant (PMFQR)Ent infections revealed that there are genetic and geospatial community links to MDR in our Chicago pediatric population [15]. Here, we report that CTX-M-9-grp-Ent infections in children are similarly linked to geographic location and that acquisition of CTX-M-9-grp-Ent in children demonstrates environmental influences and originates in the community.

\section{METHODS}

\section{Study Setting}

Hospital A contains a 115-bed children's hospital, which includes pediatric and psychiatric wards; a mother-newborn infant unit; and cardiac, neonatal, and pediatric intensive-care units (CICU, NICU, and PICU), located within a tertiary care academic medical center. Hospital $\mathrm{B}$ is a freestanding children's academic medical center comprised of 288 beds and provides quaternary care services, including bone marrow and organ transplantation. Hospital C is a 125-bed children's hospital within an academic medical center, has newborn infant and general pediatrics wards, and a NICU and PICU. The participating centers are all located within metropolitan Chicago.

\section{Descriptive Study Design}

\section{Study Population}

Patients included in this study were children $<21$ years of age who were identified because they possessed extended-spectrum cephalosporin (ceftriaxone, ceftazidime, cefotaxime)-resistant (ESC-R) Enterobacteriaceae growing in clinical cultures and were suspected to harbor a transmissible bla gene conferring cephalosporin resistance. Infections diagnosed between 1 January 2011 and 31 December 2016 were included in the analysis; only the first infection per patient was included. Study approval was obtained from the institutional review boards of the participating institutions, and the need for informed consent was waived. All procedures followed were in accordance with institutional and national ethical standards, and the study was conducted in accordance with the Helsinki Declaration of 1964 and its later amendments.

\section{Antibiotic Susceptibility Testing in Enterobacteriaceae Isolates}

The clinical microbiology laboratories of Hospitals A-C phenotypically analyzed ESC-R isolates by the Vitek 2 microbial identification system (bioMérieux, Athens, GA) or via the MicroScan WalkAway system (Beckman Coulter, Brea, CA). Following Clinical and Laboratory Standards Institute (CLSI) guidelines, one or more of the following antimicrobials, aztreonam, ceftriaxone, ceftazidime, cefotaxime, or cefpodoxime, were used to screen for ESBL production [16]. Techniques used to confirm ESBL production included automated instruments or disk diffusion assays (BBL; Becton, Dickinson and Co., Sparks, MD) to measure cefotaxime and ceftazidime susceptibility in the presence and absence of clavulanic acid. An increase in measure of a disk zone diameter of $>5 \mathrm{~mm}$ or a fourfold reduction in the minimum inhibitory concentration (MIC) of 
cefotaxime and ceftazidime in the presence of clavulanic acid served as confirmation of the ESBL phenotype [16].

\section{Molecular Analysis}

\section{Beta-Lactam Resistance Determinants}

Genomic DNA from Enterobacteriaceae isolates was purified from isolates confirmed with an ESBL phenotype (DNeasy blood and tissue kit, Qiagen, Inc. Valencia, CA). DNA Microarray (Check-MDR CT101 and CT103XL; CheckPoints, Wageningen, The Netherlands) and polymerase chain reaction (PCR) were performed to assess and confirm the presence of bla genes in isolates as previously described and according to manufacturer protocol [13, 17]. Isolates found to be positive solely for beta-lactamase genes belonging to CTX-M-9-grp were included in the analysis.

\section{Nomenclature and Characterization}

A well-established multiplex PCR-based method was used to assign E. coli to one of four phylogenetic groups (A, B1, B2, and D) [18]. Multi-locus sequence typing (MLST) [Pasteur website (http:// www.pasteur.fr/recherche/genopole/PF8/mlst/)] and DNA sequencing identified sequence types and PCR distinguished bla $_{\text {CTX-M-9-grp }}$ alleles in the ESBL-producing strains of E. coli and Klebsiella species as previously described $[13,19,20]$.

\section{Analytic Study Design}

A retrospective case-control study design was used to assess factors associated with infection due to isolates in which we had detected a bla $a_{\text {CTX-M-9-grp }}$ gene. Children serving as control subjects were identified using hospital electronic laboratory records (ELRs) and were matched 3:1 to the cases by age range, hospital, and specimen source, a design previously described $[15,21]$. Only children diagnosed with clinical infections due to Enterobacteriaceae susceptible to extended-spectrum cephalosporins (ESC-S) were included, as determined by study investigator case review and/or using standard criteria defined by the CDC National Healthcare Safety Network [22].

\section{Covariates}

We analyzed several variables as potential factors associated with CTX-M-9-grp-Ent infection based on known associations for ESBL-producing Enterobacteriaceae acquisition in adults and children, including (1) demographics (age, gender, race/ethnicity); (2) comorbid conditions (as defined by ICD-9/ICD-10 codes); (3) recent inpatient and outpatient healthcare exposures, including hospitalization and/or procedures in the previous 30 days; (4) antibiotic exposures in the 40 days prior to culture [21]; (5) presence, number, and type of invasive medical devices; and (6) patient residence in the Chicago area as assessed by dividing the metropolitan area into seven regions using zip code level data, which included Chicago proper and its suburban areas (i.e., North Side and North suburbs, South Side and South suburbs, etc.). An eighth region was included for patients residing in other states or other parts of Illinois.

\section{Statistical Analysis}

To adjust for confounding at the design stage, one case with CTX-M-9 infection was matched to three controls with antibiotic-sensitive infections by the demographic variables of interval age range $(0-1,1-5,6-12,>13$ years of age), infection source, and hospital, as matched in previously published studies [14, 15], to account for inter- and intra-facility variability in patient population and levels of care provided by the medical centers. The groups were examined for differences using chi-squared tests for categorical variables and analysis of variance for continuous variables. For the categorical variable region of residence, the West region was used as the control region. When significant differences were discovered, Fisher's exact and Wilcoxon rank sum tests were analyzed as appropriate. $P<0.05$ was considered statistically significant unless otherwise specified. Potential confounders were included in a multivariable model if associated with the outcome in bivariate analysis at $P<0.10$; they were retained if they changed the OR by $\geq 10 \%$ or had a $P$ value $<0.05$. Potential interactions 
among significant exposure variables were assessed by including an interaction term in the regression model, and statistical significance was determined with the use of the likelihood ratio test [15]. No interaction terms were found to be significant. Due to complexity of the model, sample size, and number of variables, stepwise multiple logistic regression was used to assess the multivariable relationship between the covariates and the groups. Briefly, starting with a null model where each variable was considered univariately, the variable with the smallest $P$ value was added to the model and the remaining variables were reassessed one at a time. The procedure was repeated until there were no more statistically significant covariates $(P<0.05)$. A multivariable logistic regression model was used to examine all associated factors together. The parsimonious model selected by stepwise variable selection was considered the final model and included significant covariates from the stepwise selection process and CTX-M-9 Ent infection as the dichotomous outcome variable. All analyses were performed in SAS 9.4 (SAS Institute, Cary, NC, USA).

\section{RESULTS}

Of 356 pediatric Enterobacteriaceae isolates found to harbor 350 bla genes between 2011 and 2016, $44(12.4 \%)$ were positive by DNA microarray and PCR amplification solely for bla $_{\text {CTX-M-9-grp }}$ genes. Most (40; 91\%) CTX-M-9grp-Ent infections and a significantly lesser fraction $(78 ; 59 \%)$ of ESC-S infections were E. coli, $P<0.001$ (Table 1). Urine was the most common source of CTX-M-9-grp-Ent (70\%). The antimicrobial susceptibility testing results of CTX-M-9-grp-Ent are summarized in Table 2. The highest retained susceptibility was to amikacin, carbapenems, and piperacillin-tazobactam. Multi-drug resistance (resistance to $\geq 3$ antibiotic classes) was found in $77 \%$ of case isolates.

\section{Molecular Analysis}

Of E. coli, $60 \%$ belonged to phylogenetic group $\mathrm{D}$; the rest belonged to $\mathrm{B} 2$. Both are virulent
Table 1 Characteristics of CTX M-9-grp extended-spectrum beta-lactamase (ESBL)-producing Enterobacteriaceae and ESC-sensitive Enterobacteriaceae (ESC-S)

\begin{tabular}{lll}
\hline & $\begin{array}{l}\text { CTX M-9-grp } \\
\text { ESBL }(\boldsymbol{n}=\mathbf{4 4})\end{array}$ & $\begin{array}{l}\text { ESC-S } \\
(\boldsymbol{n}=\mathbf{1 3 2})\end{array}$ \\
\hline Species, $n(\%)$ & & \\
Klebsiella pneumoniae & $3(7)$ & $78(17)$ \\
Escherichia coli & $40(91)$ & $21(16)$ \\
Enterobacter cloacae & $1(2)$ & $10(7)$ \\
Other Enterobacteriaceae & $0(0)$ & $95(72)$ \\
Source, $n$ (\%) & & $20(15)$ \\
Urine & $31(70)$ & $12(9)$ \\
Respiratory & $7(16)$ & $5(4)$ \\
Blood & $4(9)$ & $2(5)$ \\
Other & &
\end{tabular}

$E S C$ extended-spectrum cephalosporin

${ }^{\text {a }}$ Other includes wound, abscess, abdominal, and peritoneal sources

phylogroups associated with extra-intestinal pathogenic E. coli infections. Of the CTX-M-9group, the most common subtypes were CTXM-14 (61\%) and CTX-M-27 (34\%). MLST revealed diverse strain types. Of the 10 ST types associated with 40 CTX-M-9 E. coli, 25\% were ST8; ST43/ST131 and ST506 were the next most common (20\% and 15\%, respectively). Two novel $E$. coli strain types were also discovered to carry $b l a_{\text {CTX-M-9-grp }}$ (data not shown).

\section{Analysis of Factors Associated with CTX- M-9 Ent Infection}

The 44 cases of CTX-M-9-grp-Ent infection were matched to 132 ESC-S controls. On bivariate analysis (Table 3 ) factors associated with higher likelihood of CTX-M-9-grp-Ent infection compared with ESC-S controls included: infection with $E$. coli, diagnosis in an outpatient clinic, absence of recent health care, residence in the South or Southwest regions (comprising South and Southwest Chicago and associated suburbs), and race "other" (non-White, non-Black, non-Hispanic). Children with CTX-M-9-grp-Ent 


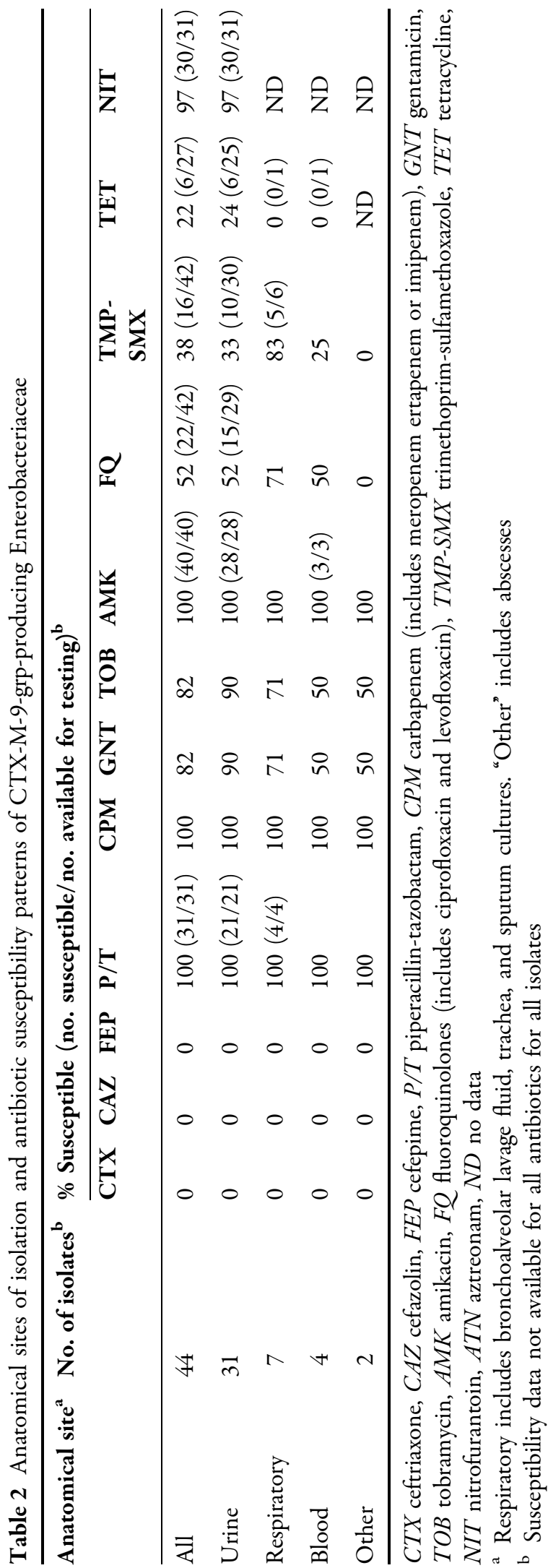

infections were less likely than controls to have infection with Enterobacter sp., diagnosis in the inpatient, non-ICU setting (i.e., general pediatric wards), residence in the Northwest region (comprised of Northwestern Chicago and the Northwestern suburbs), presence of a central venous line, respiratory comorbidities, and recent outpatient health care (including outpatient procedures).

During model building stages, we did not find evidence of a significant effect modification or significant confounding; therefore, additional covariates were not added back to the final model after the stepwise selection process was completed, and the simplest model with significant covariates was included in the final regression model.

In the multivariable regression analysis (Table 4), factors associated with CTX-M-9-grpEnt infection included having infection due to E. coli (OR 7.4; 95\% CI 2.4, 27.2; $P=0.001$ ), being of "other" race or ethnicity (OR 7.4; 95\% CI 2.4, 28.2; $P=0.002)$, and being diagnosed in the outpatient clinic setting (OR 4.5; 95\% CI $1.7,12.3 ; P=0.003)$.

Remarkably, among children with Enterobacteriaceae infections, those residing in the South region of Chicago demonstrated more than six times the odds of having a CTX-M-9grp-Ent infection compared with those living in the reference West Chicago region (OR 6.7; 95\% CI 1.1, 37.9; $P=0.03$ ) after controlling for race, infecting organism, and healthcare setting. In contrast, for children who resided in the Northwest region, there was an $81 \%$ decrease in the odds of CTX-M-9-grp-Ent infection compared with those residing in the reference West Chicago region (OR 0.19; 95\% CI 0.04, 0.90; $P=0.03)$.

\section{DISCUSSION}

Our analysis is the first multi-center study to assess the clinical and molecular epidemiology of CTX-M-9-type ESBL-producing Enterobacteriaceae infections in children located in an urban setting. We found that E. coli was the most common infecting organism associated

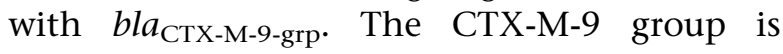


Table 3 Bivariate analysis of demographics and factors associated with CTX-M-9-grp Enterobacteriaceae infections in children

\begin{tabular}{|c|c|c|c|}
\hline Characteristic $^{a}$ & CTX-M-9-grp Ent infection & ESC-S Ent infection ${ }^{b}$ & $P$ value \\
\hline Patient & $n=44$ & $n=132$ & \\
\hline Age & $4.14(0-20.8)$ & $4.48(0-20.9)$ & 0.11 \\
\hline Female & $29(65.9)$ & $92(69.7)$ & 0.64 \\
\hline Race, White & $15(34.1)$ & $38(28.8)$ & 0.51 \\
\hline Race, Black & $3(6.8)$ & $29(22.0)$ & 0.03 \\
\hline Race, Hispanic & $15(34.1)$ & $59(44.7)$ & 0.22 \\
\hline Race, non-White, non-Black, non-Hispanic & $11(25)$ & $6(4.6)$ & $<0.0001$ \\
\hline Location at diagnosis & & & $<0.0001$ \\
\hline Inpatient, non-ICU & $7(15.9)$ & $42(31.8)$ & 0.04 \\
\hline Outpatient clinic & $16(36.4)$ & $10(7.6)$ & $<0.001$ \\
\hline Emergency room & $9(20.5)$ & $23(17.4)$ & 0.65 \\
\hline Pediatric ICU & $9(20.5)$ & $39(29.6)$ & 0.24 \\
\hline Neonatal ICU & $3(6.8)$ & $18(13.6)$ & 0.22 \\
\hline Recent CTX ${ }^{\mathrm{e}}$ & $9(15.9)$ & $33(25)$ & 0.21 \\
\hline Recent $F Q^{f}$ & $3(6.8)$ & $4(3.0)$ & 0.27 \\
\hline Recent TMP/SMX & $5(11.4)$ & $18(13.9)$ & 0.68 \\
\hline Region of residence ${ }^{c}$ & & & 0.01 \\
\hline Recent health care & & & 0.01 \\
\hline Inpatient care & $11(25)$ & $34(25.8)$ & 0.92 \\
\hline Outpatient care $^{\mathrm{d}}$ & $10(22.7)$ & $59(44.7)$ & 0.01 \\
\hline No recent care & $23(52.3)$ & $39(29.6)$ & 0.006 \\
\hline Foreign body present & $19(43.2)$ & $75(56.8)$ & 0.12 \\
\hline Multiple foreign bodies present & $13(29.6)$ & $49(37.1)$ & 0.36 \\
\hline Central venous line comorbidities & $8(18.2)$ & $44(33.3)$ & 0.03 \\
\hline Cardiovascular & $7(15.9)$ & $29(22.0)$ & 0.39 \\
\hline Endocrine & $2(4.6)$ & $9(7.0)$ & 0.73 \\
\hline Gastrointestinal & $12(27.3)$ & $32(24.2)$ & 0.69 \\
\hline Genitourinary & $14(26.4)$ & $37(28.0)$ & 0.82 \\
\hline Hematology & $6(13.6)$ & $20(15.2)$ & 0.81 \\
\hline Neurology & $14(31.8)$ & $47(35.6)$ & 0.65 \\
\hline Respiratory & $8(18.2)$ & $47(35.6)$ & 0.03 \\
\hline
\end{tabular}


Table 3 continued

\begin{tabular}{|c|c|c|c|}
\hline$\overline{\text { Characteristic }^{a}}$ & CTX-M-9-grp Ent infection & ESC-S Ent infection ${ }^{b}$ & $P$ value \\
\hline Renal & $13(29.6)$ & $41(31.1)$ & 0.85 \\
\hline Multiple comorbidities & $18(40.9)$ & $63(47.0)$ & 0.49 \\
\hline $\begin{array}{l}\text { a } \text { Values represent } n \text { (\% } \\
\text { b } \text { ESC-S Ent infections } \\
\text { losporin antibiotics } \\
\text { c Region of residence in } \\
\text { d } \text { Outpatient care incluc } \\
\text { e } \\
\text { e Abbreviation CTX in } \\
\text { f } \text { FQ, fluoroquinolones } \\
\text { g } \text { TMP-SMX, trimetho }\end{array}$ & $\begin{array}{l}\text { ated } \\
\text { fections due to Enterobacteriace } \\
\text { neighboring suburbs } \\
\text { ine child care visits and outpatie } \\
m \text { cephalosporins (ceftriaxone, c } \\
\text { loxacin) }\end{array}$ & $\begin{array}{l}\text { sensitive to extended-spe } \\
\text { procedures } \\
\text { zidime, cefotaxime, cefep }\end{array}$ & um cepha \\
\hline
\end{tabular}

Table 4 Multivariable analysis of factors associated with CTX-M-9-grp Enterobacteriaceae infections in children

\begin{tabular}{|c|c|c|c|}
\hline Associated factors with CTX-M-9-grp Ent infection ${ }^{\mathrm{a}, \mathrm{c}}$ & OR & $95 \% \mathrm{CI}$ & $P$ value \\
\hline Infection with $E$. coli & 7.4 & $2.4,27.2$ & 0.001 \\
\hline Home residence in South region (South Chicago and South suburbs) ${ }^{b}$ & 6.7 & $1.1,37.9$ & 0.03 \\
\hline Race not White, Black or Hispanic & 7.4 & $2.4,28.2$ & 0.002 \\
\hline Outpatient clinic location at time of infection diagnosis & 4.5 & $1.7,12.3$ & 0.003 \\
\hline Home residence in Northwest region (NW Chicago and NW suburbs) & 0.19 & $0.04,0.90$ & 0.03 \\
\hline
\end{tabular}

thought to be the second most commonly community acquired ESBL genes in Enterobacteriaceae worldwide, and, similar to the highrisk clonal ST131 CTX-M-15 producing E. coli, are reported in people without significant healthcare exposure and are associated with multiple antibiotic gene cassettes leading to MDR $[3,9]$. However, unlike the association of the CTX-M-1 group with ST131, we found that bla $a_{\text {CTX-M-9-grp }}$ genes are carried by multiple strain types, including the identification of novel STs associated with pediatric infection.

Because of multiple associated strain types carrying $b l a_{\text {CTX-M-9-grp }}$ genes, we hypothesized that significant horizontal gene transfer is occurring between genera. This observation is concerning as children once colonized with MDR Enterobacteriaceae can remain colonized for months to years, and young children (median age 4.1 years) were carrying organisms with multiple plasmids, capable of rapid dissemination and spread [13, 23, 24]. Additionally, we found novel ST types carrying $b l a_{\text {CTX-M-9-grp }}$ genes. This portends a troublesome epidemiology suggesting the acquisition of these genes may be occurring in commensal or previously non-pathogenic strains of Enterobacteriaceae.

In our study, most (77\%) CTX-M-9-grp-Ent isolates were phenotypically MDR. While the highest retained antibiotic susceptibility included piperacillin-tazobactam, clinicians should be cautioned in its use for serious, invasive infections (e.g., bacteremia). Recent randomized clinical trials have demonstrated inferiority of piperacillin-tazobactam to carbapenem therapy when used to treat ESC-R Ent bloodstream infections (including ESBL producers) and describe worse patient outcomes associated with piperacillin-tazobactam use as definitive therapy [25]. Of additional concern, susceptibility to oral agents was significantly reduced 
leaving few oral options for step-down therapy for children (Table 2). The sole oral exception was nitrofurantoin ( $97 \%$ sensitivity), which can be used to treat uncomplicated lower urinary tract infection but should not be used for complicated, invasive infections or acute pyelonephritis because of the inability of the drug to attain therapeutic levels in the renal parenchyma and bloodstream [26].

The residential differences for children infected with CTX-M-9-grp-Ent compared with children infected with antibiotic-sensitive strains were striking. We found a substantial increase in odds of infection with the resistant strains in the South Chicago region and a significant decrease in odds of infection in the Northwest Chicago region. Our study included three major pediatric centers, none of which are located in the "high-risk" South region, yet all three centers diagnosed and treated patients with CTX-M-9-grp-Ent infections from these high-risk regions. The reservoirs associated with acquisition of CTX-M-9-grp-Ent in these regions are currently undefined.

Of additional concern, in a separate study of plasmid-mediated fluoroquinolone-resistant (PMFQR) Ent infections in children between 2011 and 2014, we found a substantial increase in odds of PMFQR Ent infection in the Southwest region, while in the Downtown region (comprising the downtown Chicago area, near North side, Chicago loop, and North Chicago), there was a significant decrease in the likelihood of PMFQR Ent infection [15]. These PMFQR Ent "high-risk" and "low-risk" regions neighbor the "high-risk" South region and "lowrisk" Northwest region in the current study. The Southwest region was found to be associated with an increase in the odds of CTX-M-9-grpEnt infection on bivariate analysis in this study, though this did not reach statistical significance on multivariable analysis once controlling for other variables.

Furthermore, we found that children with CTX-M-9-grp-Ent infection were significantly more likely to present in the outpatient clinic setting compared with those with antibiotic-susceptible Ent infections. This is surprising and striking as most infections caused by MDROs in the US are linked to healthcare settings [27]. While the majority of infections were associated with virulent extra-intestinal-pathogenic E. coli phylogenetic groups ( $B$ and D), these data suggest that CTX-M-9-grp-Ent infections in children are being acquired in the community and may be less severe at presentation as children presented more often in outpatient clinics. This is in stark contrast to the epidemiology of MDR Enterobacteriaceae infections reported in adults in Chicago, where acquisition has been strongly associated with residence in long-term care facilities and the interfacility transfer of patients [28].

The higher likelihood of CTX-M-9-grp-Ent infection in those of non-White, non-Black, and non-Hispanic race was an additional association discovered on multivariable analysis. This risk was statistically independent of residence, and only one of the children located in the "high risk" South region was of race "other," supporting the independence of the two risk factors for infection. We were unable to gather further data on "other" race or ethnicity because of the retrospective nature of the study, and we did not have travel data for the majority of children, which are important limitations. It is well described that travel to certain countries can be associated with high rates of ESBL Ent acquisition, particularly in South and Southeast Asia [29]. It is also well described that there is an increased risk of colonization when residing in a household with a family member with history of ESBL Ent or who acquired such a strain during travel to an endemic region [30].

Environmental influences originating in the community could include higher risks in certain populations due to specific exposures to foods, animals, water sources, fertilizer, soil, and vegetation [31]. We did not have data on companion pets for the majority of children and were unable to examine this risk factor, though pets may play a significant role in human acquisition of antibiotic-resistant bacteria [32]. Lastly, we did not find overall differences in a general comparison of socioeconomic status of the "high-risk" South region and neighboring regions such as the Southwest and West regions using regional zip codes and Illinois census data.

We recognize the limitations of our study. This was a retrospective study of children with 
Enterobacteriaceae infections in a single metropolitan area, which may affect generalizability to other regions. While selection bias may result from small sample sizes, the pooling of multicentered data from institutions of differing types in the third largest US metropolitan area and serving diverse populations potentially lessens this bias. Moreover, our sample size is consistent with the overall low prevalence of these MDR Enterobacteriaceae children in the majority of the US (1-3\%) including Chicago and the Midwest region [33]; however, it should be noted that national trends indicate an increase in the incidence and prevalence of pediatric MDR Enterobacteriaceae over the last decade, an emerging threat that needs further assessment $[5,34]$. Finally, we did not compare these CTX-M-9-grp-Ent infections with those due to other bla genes; however, future comparative studies are planned.

\section{CONCLUSION}

We describe, for the first time, community origins of CTX-M-9-grp Enterobacteriaceae in children and the impact of residence on infection in children located in the same geographic area. The reservoirs associated with CTX-M-9grp-Ent infections remain undefined. Future studies must focus on environmental influences associated with regional acquisition. "Silent dissemination" of community-acquired MDR Enterobacteriaceae is likely occurring in children outside of healthcare settings. Dedicated programs on a local, national, and global scale must focus on children and halting of spread of these dangerous pathogens.

\section{ACKNOWLEDGEMENTS}

We gratefully acknowledge the contribution of the late Dr. Paul Schreckenberger to this work. We thank the microbiology laboratories of the participating institutions for providing isolates for this study. We thank Laura Rojas-Coy for contributions in the CTX-M primer design for PCR amplification. We thank Kendrick Reme and Lynika Strozier of the Logan Laboratory and
Pamela Hagen, Jane Stevens, Joyce Houlihan, Kathleen McKinley, Violeta Rekasiu, Cindy Bethel, and Donna Carter of participating institutions for collection, shipping, and cultivation of organisms. We thank the team of curators of the Institut Pasteur MLST and whole-genome MLST databases for curating the data and making them publicly available at http://bigsdb.web.pasteur.fr/. The content is solely the responsibility of the authors and does not necessarily represent the official views of the National Institutes of Health or the Department of Veterans Affairs. This work was previously published as a preprint: https://www. biorxiv.org/content/early/2018/09/20/416016.

Funding. This work, including the efforts of Latania K. Logan and associated article processing charges, was funded by the National Institute of Allergy and Infectious Diseases, National Institutes of Health (NIH) (K08AI112506). This work, including the efforts of Robert A. Bonomo, was funded by the National Institute of Allergy and Infectious Diseases, National Institutes of Health (NIH) (R01AI072219, R01AI063517, and R01AI100560). Robert A. Bonomo is also supported by the Department of Veterans Affairs Research and Development under award number I01BX001974, VISN 10 Geriatrics Research, Education and Clinical Center.

Authorship. All named authors meet the International Committee of Medical Journal Editors (ICMJE) criteria for authorship for this article, take responsibility for the integrity of the work as a whole, and have given their approval for this version to be published.

Disclosures. Latania K. Logan, Rachel L. Medernach, T. Nicholas Domitrovic, Jared R. Rispens, Andrea M. Hujer, Nadia K. Qureshi, Steven H. Marshall, David C. Nguyen, Susan D. Rudin, Xiaotian Zheng, Sreenivas Konda, Robert A. Weinstein, and Robert A. Bonomo have nothing to disclose. At the time of the study, Jared R. Rispens' affiliations were Pediatrics, Rush University Medical Center, Chicago, IL, USA; Medicine, Rush University Medical Center, Chicago, Illinois, USA. Jared R. Rispens' 
current affiliation is Centers for Disease Control and Prevention, Atlanta, GA, USA.

Compliance with Ethics Guidelines. Study approval was obtained from the institutional review boards of the participating institutions and the need for informed consent was waived. All procedures followed were in accordance with institutional and national ethical standards, and the study was conducted in accordance with the Helsinki Declaration of 1964 and its later amendments.

Data Availability. The data sets during and/ or analyzed during the current study are available from the corresponding author on reasonable request.

Open Access. This article is distributed under the terms of the Creative Commons AttributionNonCommercial 4.0 International License (http:// creativecommons.org/licenses/by-nc/4.0/), which permits any noncommercial use, distribution, and reproduction in any medium, provided you give appropriate credit to the original author(s) and the source, provide a link to the Creative Commons license, and indicate if changes were made.

\section{REFERENCES}

1. Logan LK, Weinstein RA. The epidemiology of carbapenem-resistant enterobacteriaceae: the impact and evolution of a global menace. J Infect Dis. 2017;215(suppl_1):S28-36.

2. Medernach RL, Logan LK. The growing threat of antibiotic resistance in children. Infect Dis Clin $\mathrm{N}$ Am. 2018;32(1):1-17.

3. Lukac PJ, Bonomo RA, Logan LK. Extended-spectrum beta-lactamase-producing Enterobacteriaceae in children: old foe, emerging threat. Clin Infect Dis. 2015;60(9):1389-97.

4. Logan LK, Renschler JP, Gandra S, Weinstein RA, Laxminarayan R, Centers for Disease Control, et al. Carbapenem-resistant Enterobacteriaceae in children, United States, 1999-2012. Emerg Infect Dis. 2015;21(11):2014-21.

5. Logan LK, Braykov NP, Weinstein RA, Laxminarayan R, CDC Epicenters Prevention Program.
Extended-spectrum beta-lactamase-producing and third-generation cephalosporin-resistant enterobacteriaceae in children: trends in the United States, 1999-2011. J Pediatr Infect Dis Soc. 2014;3(4):320-8.

6. Munoz-Price LS, Poirel L, Bonomo RA, Schwaber MJ, Daikos GL, Cormican M, et al. Clinical epidemiology of the global expansion of Klebsiella pneumoniae carbapenemases. Lancet Infect Dis. 2013;13(9):785-96.

7. Price LB, Johnson JR, Aziz M, Clabots C, Johnston B, Tchesnokova $\mathrm{V}$, et al. The epidemic of extendedspectrum-beta-lactamase-producing Escherichia coli ST131 is driven by a single highly pathogenic subclone, H30-Rx. MBio. 2013;4(6):e00377-13.

8. Miles-Jay A, Weissman SJ, Adler AL, Tchesnokova V, Sokurenko EV, Baseman JG, et al. Epidemiology and antimicrobial resistance characteristics of the sequence type 131-H30 subclone among extraintestinal Escherichia coli collected from US children. Clin Infect Dis. 2017;66(3):411-9.

9. Cantón R, González-Alba JM, Galán JC. CTX-M enzymes: origin and diffusion. Front Microbiol. 2012;3:110.

10. Merida-Vieyra J, De Colsa A, Castañeda YC, Barbosa PA, Andrade AA. First report of group CTX-M-9 extended spectrum beta-lactamases in Escherichia coli isolates from pediatric patients in Mexico. PLoS One. 2016;11(12):e0168608.

11. Castanheira M, Mendes RE, Jones RN, Sader HS. Changes in the frequencies of beta-lactamase genes among Enterobacteriaceae isolates in US hospitals, 2012 to 2014: activity of ceftazidime-avibactam tested against beta-lactamase-producing isolates. Antimicrob Agents Chemother. 2016;60(8):4770-7.

12. Oteo J, Diestra K, Juan C, Bautista V, Novais Â, Pérez-Vázquez $M$, et al. Extended-spectrum $\beta$-lactamase-producing Escherichia coli in Spain belong to a large variety of multilocus sequence typing types, including ST10 complex/A, ST23 complex/A and ST131/B2. Int J Antimicrob Agents. 2009;34(2): 173-6.

13. Logan LK, Hujer AM, Marshall SH, Domitrovic TN, Rudin SD, Zheng X, et al. Analysis of beta-lactamase resistance determinants in enterobacteriaceae from Chicago children: a multicenter survey. Antimicrob Agents Chemother. 2016;60(6):3462-9.

14. Logan LK, Nguyen DC, Scaggs-Huang FA, Qureshi NK, Charnot-Katsikas A, Bartlett AH, et al. A multicentered case-case-control study of factors associated with Klebsiella pneumoniae carbapenemase (KPC)-producing Enterobacteriaceae (KPC-CRE) infections in children and young adults. Pediatr 
Infect Dis J. 2019;38(6):HS2-5 (E-pub ahead of print).

15. Logan LK, Medernach RL, Rispens JR, Marshall SH, Hujer AM, Domitrovic TN, et al. Community origins and regional differences highlight risk of plasmid-mediated fluoroquinolone resistant Enterobacteriaceae infections in children. Pediatr Infect Dis J 2019;38(7) (E-pub ahead of print).

16. Clinical and Laboratory Standards Institute. Performance standards for antimicrobial susceptibility testing: twentieth informational supplement (June 2010 update). 2010:December 29, 2011. https://clsi. org/media/1469/m100s27_sample.pdf.

17. Powell EA, Haslam D, Mortensen JE. Performance of the check-points check-MDR CT103XL assay utilizing the CDC/FDA antimicrobial resistance isolate bank. Diagn Microbiol Infect Dis. 2017;88(3):219-21.

18. Bingen-Bidois $\mathrm{M}$, Clermont $\mathrm{O}$, Bonacorsi $\mathrm{S}$, Terki $\mathrm{M}$, Brahimi N, Loukil C, et al. Phylogenetic analysis and prevalence of urosepsis strains of Escherichia coli bearing pathogenicity island-like domains. Infect Immun. 2002;70(6):3216-26.

19. Diancourt L, Passet V, Verhoef J, Grimont PA, Brisse S. Multilocus sequence typing of Klebsiella pneumoniae nosocomial isolates. J Clin Microbiol. 2005;43(8):4178-82.

20. Jaureguy F, Landraud L, Passet V, Diancourt L, Frapy E, Guigon G, et al. Phylogenetic and genomic diversity of human bacteremic Escherichia coli strains. BMC Genom. 2008;9:560-2164-9-560.

21. Logan LK, Meltzer LA, McAuley JB, Hayden MK, Beck T, Braykov NP, et al. Extended-spectrum betalactamase-producing Enterobacteriaceae infections in children: a two-center case-case-control study of risk factors and outcomes in Chicago, Illinois. J Pediatr Infect Dis Soc. 2014;3(4):312-9.

22. Centers for Disease Control and Prevention (CDC). The National Healthcare Safety Network (NHSN) patient safety component manual. 2017. https:// www.cdc.gov/nhsn/pdfs/validation/2017/pcsmanual_ 2017.pdf

23. Carattoli A, Bertini A, Villa L, Falbo V, Hopkins KL, Threlfall EJ. Identification of plasmids by PCR-based replicon typing. J Microbiol Methods. 2005;63(3): 219-28.

24. Zerr DM, Qin X, Oron AP, Adler AL, Wolter DJ, Berry JE, et al. Pediatric infection and intestinal carriage due to extended-spectrum-cephalosporinresistant Enterobacteriaceae. Antimicrob Agents Chemother. 2014;58(7):3997-4004.
25. Harris PN, Tambyah PA, Lye DC, Mo Y, Lee TH, et al. Effect of piperacillin-tazobactam vs meropenem on 30-day mortality for patients with E. coli or Klebsiella pneumoniae bloodstream infection and ceftriaxone resistance: a randomized clinical trial. JAMA. 2018;13(10):984-94.

26. Beetz R, Westenfelder M. Antimicrobial therapy of urinary tract infections in children. Int J Antimicrob Agents. 2011;38:42-50.

27. Sievert DM, Ricks P, Edwards JR, Schneider A, Patel J, Srinivasan A, et al. Antimicrobial-resistant pathogens associated with healthcare-associated infections summary of data reported to the National Healthcare Safety Network at the Centers for Disease Control and Prevention, 2009-2010. Infect Control Hosp Epidemiol. 2013;34(1):1-14.

28. Snitkin ES, Won S, Pirani A, Lapp Z, Weinstein RA, Lolans $\mathrm{K}$, et al. Integrated genomic and interfacility patient-transfer data reveal the transmission pathways of multidrug-resistant Klebsiella pneumoniae in a regional outbreak. Sci Transl Med. 2017. https:// doi.org/10.1126/scitranslmed.aan0093.

29. Kuenzli E, Jaeger VK, Frei R, Neumayr A, DeCrom S, Haller $S$, et al. High colonization rates of extendedspectrum $\beta$-lactamase (ESBL)-producing Escherichia coli in Swiss travellers to South Asia-a prospective observational multicentre cohort study looking at epidemiology, microbiology and risk factors. BMC Infect Dis. 2014;14(1):528.

30. Haverkate MR, Platteel TN, Fluit A, Stuart JC, Leverstein-van Hall M, Thijsen S, et al. Quantifying within-household transmission of extended-spectrum $\beta$-lactamase-producing bacteria. Clin Microbiol Infect. 2017;23(1):46.e1-7.

31. Silbergeld EK, Graham J, Price LB. Industrial food animal production, antimicrobial resistance, and human health. Annu Rev Public Health. 2008;29:151-69.

32. Grasselli E, François P, Gutacker M, Gettler B, Benagli C, Convert $\mathrm{M}$, et al. Evidence of horizontal gene transfer between human and animal commensal Escherichia coli strains identified by microarray. FEMS Immunol Med Microbiol. 2008;53(3):351-8.

33. Zerr DM, Weissman SJ, Zhou C, Kronman MP, Adler $\mathrm{AL}$, Berry JE, et al. The molecular and clinical epidemiology of extended-spectrum cephalosporin-and carbapenem-resistant Enterobacteriaceae at 4 US pediatric hospitals. J Pediatr Infect Dis Soc. 2017;6:366-75.

34. Meropol SB, Haupt AA, Debanne SM. Incidence and outcomes of infections caused by multidrug-resistant enterobacteriaceae in children, 2007-2015. J Pediatr Infect Dis Soc. 2017;7(1):36-45. 